\title{
Primary prevention with lipid lowering drugs and long term risk of vascular events in older people: population based cohort study
}

\author{
Annick Alpérovitch,,,2 Tobias Kurth,1,2 Marion Bertrand,, ,2 Marie-Laure Ancelin,3,4 \\ Catherine Helmer, ${ }^{1,2}$ Stéphanie Debette, ${ }^{1,2}$ Christophe Tzourio ${ }^{1,2}$
}

1INSERM, U897-Epidemiology and Biostatistics, Bordeaux,

France

2Université de Bordeaux Bordeaux, France

3INSERM, U1061, Montpellier, France

«Université de Montpellier I, Montpellier, France

Correspondence to: Christophe Tzourio, Inserm U897, Université de Bordeaux 146 rue Léo Saignat - Case 11, 33076 Bordeaux cedex, France christophe.tzourio@u-bordeaux.fr Additional material is published online only. To view please visit the journal online (http://dx.doi. org/10.1136/bmj.h2335)

Cite this as: $B M J$ 2015;350:h2335 doi: 10.1136/bmj.h2335

Accepted: 23 March 2015

\begin{abstract}
OBJECTIVE

To determine the association between use of lipid lowering drugs (statin or fibrate) in older people with no known history of vascular events and long term risk of coronary heart disease and stroke.

DESIGN

Ongoing prospective population based cohort study recruited in 1999-2000, with five face-to-face examinations.

\section{SETTING}

Random sample of community dwelling population aged 65 years and over, living in three French cities (Bordeaux, Dijon, Montpellier).
\end{abstract}

\section{PARTICIPANTS}

7484 men and women (63\%) with mean age 73.9 years and no known history of vascular events at entry. Mean follow-up was 9.1 years.

\section{MAIN OUTCOME MEASURES}

Adjusted hazard ratios of coronary heart disease and stroke in baseline lipid lowering drug users compared with non-users, calculated using multivariable Cox proportional hazard models adjusted for numerous potential confounding factors. Hazard ratios were estimated for use of any lipid lowering drug and for statin and fibrate separately.

RESULTS

Lipid lowering drug users were at decreased risk of stroke compared with non-users (hazard ratio 0.66, $95 \%$ confidence interval 0.49 to 0.90$)$; hazard ratios for stroke were similar for statin $(0.68,0.45$ to1.01) and

\section{WHAT IS ALREADY KNOWN ON THIS TOPIC}

Randomised controlled trials have established the efficacy of lipid lowering drugs for secondary and primary prevention of cardiovascular events (coronary heart disease, stroke, or both) in the population aged 50 to 70 years

In clinical trial participants, the effect of lipid lowering drugs is greater for coronary heart disease than for stroke events and risk reduction is greater for statins than for fibrates

New guidelines do not support lipid lowering treatment in people aged over 75 years without clinical atherosclerotic disease, but lipid lowering drugs are largely used for primary prevention in the older age groups

\section{WHAT THIS STUDY ADDS}

Use of lipid lowering drugs was associated with a 30\% lower risk of stroke (compared with non-users) during a mean follow-up of 9 years in a large population based cohort (mean age 74 years) without known history of vascular disease Reduction in risk of stroke was similar in statin users and fibrate users If replicated, the study results suggest that lipid lowering drugs might be considered for the prevention of stroke in older populations

fibrate $(0.66,0.44$ to 0.98$)$. No association was found between lipid lowering drug use and coronary heart disease (hazard ratio 1.12, 0.90 to 1.40). Analyses stratified by age, sex, body mass index, hypertension, systolic blood pressure, triglyceride concentrations, and propensity score did not show any effect modification by these variables, either for stroke or for coronary heart disease.

\section{CONCLUSION}

In a population based cohort of older people with no history of vascular events, use of statins or fibrates was associated with a $30 \%$ decrease in the incidence of stroke.

\section{Introduction}

In high income countries, a growing proportion of vascular events occur in the oldest people. According to the French national mortality statistics for 2010, people aged 85 years and over accounted for $43 \%$ of deaths from coronary heart disease and $49 \%$ of deaths from stroke. In contrast, participants in most randomised controlled trials testing cardiovascular drugs are predominantly under the age of 70 years. ${ }^{1}$ Therefore, the benefit of these drugs in the oldest people remains uncertain. Randomised trials support the use of hydroxymethyl glutaryl coenzyme A reductase inhibitors (statins) for reducing the incidence of major cardiovascular and cerebrovascular events in people with a history of cardiovascular disease, including those aged 65 years and over. ${ }^{2-5}$ However, evidence of the benefit of statin treatment for primary prevention is limited, especially in older people. Regarding fibrates, the other main class of lipid lowering drugs, very few trials support their efficacy for the primary prevention of cardiovascular events. ${ }^{6-9}$

New guidelines about cholesterol management based on evidence from randomised controlled trials do not recommend statin treatment in people over 75 years of age without clinical atherosclerotic disease, whereas, in real life, statins are commonly prescribed to older people without clinical evidence of atherosclerosis. ${ }^{1011}$ Avoiding chronic use of unnecessary drugs is important in older people, who often take many drugs and are at high risk of adverse effects. Because of the limited trial based data on the effect of lipid lowering drugs for primary prevention of vascular events in older people, observational studies in this age group can be valuable. We aimed to study the association between use of lipid lowering drugs and the risk of incident cardiovascular events in a cohort study of 7484 community living people aged 65 years or over at entry with a mean follow-up of nine years. 


\section{Methods}

Study population

The Three-City study is a prospective study aiming to assess the association between vascular diseases and risk of dementia. The detailed study protocol has been previously described. ${ }^{12}$ The Three-City cohort is composed of non-institutionalised people aged 65 years and over, randomly selected from electoral rolls of three cities in France (Bordeaux (south west), Dijon (north east), and Montpellier (south east), who agreed to participate in the study and signed an informed consent form.

Between March 1999 and March 2001, 9294 people were enrolled. A total of 1439 participants were not eligible for the study described here, as they reported a history of coronary heart disease $(n=1017)$, stroke $(n=330)$, or both vascular events $(n=92)$ at baseline. Participants treated with lipid lowering drugs other than statins or fibrates (for example, bile acid sequestrants) $(\mathrm{n}=113)$ were also excluded. Among the 7742 remaining participants, 258 (3.3\%) were lost to follow-up, leaving a study sample of 7484 participants.

Face-to-face examinations took place every two years during follow-up. Trained nurses and psychologists conducted interviews and made physical and cognitive measurements at the participant's home and at the study centre. Data collection included sociodemographic characteristics (education, occupation, and income), lifestyle (smoking, drinking, and food frequency questionnaire), assessment of disability (Instrumental Activities of Daily Living scale), global cognitive functioning (Mini Mental State examination) and depression (Centre for Epidemiologic Studies-Depression scale), and recording of height and weight. ${ }^{131415}$ Past history of cardiovascular disease included a history of coronary heart disease, stroke, arrhythmia, and peripheral artery disease. Blood pressure was measured twice after five minutes' rest in a seated position with an electronic device (OMRON M6; OMRON Healthcare, Kyoto, Japan). Hypertension was defined as a systolic blood pressure $140 \mathrm{~mm} \mathrm{Hg}$ or above, a diastolic blood pressure $90 \mathrm{~mm} \mathrm{Hg}$ or above, or the use of antihypertensive treatment. At baseline, blood was collected after overnight fasting. Lipid concentrations (total cholesterol, high density lipoprotein cholesterol, low density lipoprotein cholesterol, and triglycerides) and glycaemia were measured centrally. Diabetes was defined as use of antidiabetic drugs or a fasting blood glucose of $7 \mathrm{mmol} / \mathrm{L}$ or above.

\section{Use of lipid lowering and other drugs}

At each follow-up examination, at the participants' home, interviewers collected information on all drugs used during the preceding month. Participants were asked to show their prescriptions and drug packages. Drug names were coded according to the Anatomic Therapeutic Chemical classification of the World Health Organization. ${ }^{16}$ Lipid lowering drugs (code C10A) included hydroxymethyl glutaryl coenzyme A reductase inhibitors (C10AA), fibrates (C10AB), and other drugs such as bile acid sequestrants or nicotinic acid derivatives (not considered in this study). Use of blood pressure lowering or antithrombotic drugs was also registered.

Ascertainment of vascular events during follow-up At each follow-up visit, participants or informants for deceased participants were systematically questioned about the occurrence of any severe medical event or hospital admission since the last contact. For those reporting a possible coronary heart disease or stroke event, all available clinical information was collected from hospital records, and interviews were conducted with the participant's physician, nursing home staff (for participants admitted to a nursing home during follow-up), or family. Expert panels reviewed all available clinical information and classified each event according to ICD-10 (international classification of diseases, 10th revision). Cardiac events included hospital admission for definite angina, definite myocardial infarction, definite cardiovascular death, coronary balloon dilatation, or coronary artery bypass. Brain imaging data were available for more than $80 \%$ of validated stroke cases (computerised tomography 82\%; magnetic resonance imaging 15\%) and Doppler ultrasound for 62\%. When no brain imaging was available, the diagnosis was based on signs and symptoms. Stroke was confirmed if the participant had a new focal neurological deficit of sudden onset attributable to a cerebrovascular event that persisted for more than 24 hours. The panel classified stroke as ischaemic stroke, intracerebral haemorrhage, or of unspecified type.

\section{Statistical analysis}

We described the characteristics of the cohort according to lipid lowering drug use at baseline. We compared users and non-users of lipid lowering drugs by using analysis of variance and $\chi^{2}$ tests adjusted for age, sex, and centre. We also compared users of statins and users of fibrates, and we assessed the associations between vascular events and classic vascular risk factors. We estimated the risk of vascular events related to lipid lowering drug use by using a Cox proportional hazards model with age as the timescale, and we calculated hazard ratios and their 95\% confidence intervals with participants not taking any lipid lowering drug as the reference. Modelling included testing of the proportional hazard assumption. We estimated hazard ratios for any major fatal or non-fatal vascular event (coronary heart disease or stroke) and for each type of event separately, using successively use of any lipid lowering drug, statins, and fibrates as independent variables. If a participant had multiple cardiovascular events during follow-up, we considered only the date of the first in estimating the overall risk of vascular event. We first used a simple model adjusted for sex and study centre, with age used as the timescale (model 1). The multivariable model (model 2) was further adjusted for potential confounding factors: diabetes (yes, no), body mass index $(<25,25-29, \geq 30)$, smoking (never, past, current), drinking alcohol (never, past, current), hypertension (yes, no), arrhythmia (yes, no), antithrombotic drugs (yes, no), triglyceride concentration (thirds), and low 
density lipoprotein to high density lipoprotein cholesterol ratio (thirds).

We did sensitivity analyses stratified by age $(<75$, $\geq 75)$, sex, triglyceride concentration $(<1.6 \mathrm{~g} / \mathrm{L}, \geq 1.6$ $\mathrm{g} / \mathrm{L})$, body mass index $(<27, \geq 27)$, hypertension (yes, no), and systolic blood pressure ( $<145 \mathrm{~mm} \mathrm{Hg}, \geq 145 \mathrm{~mm}$ $\mathrm{Hg}$ ) for coronary heart disease and stroke separately. We built a high dimensional propensity score for lipid lowering drug use with a logistic regression model including all the adjustment variables of the primary multivariable model plus other available variables (education, income, systolic blood pressure, diastolic blood pressure, use of antihypertensive drug, self reported diabetes, glycaemia $\geq 7 \mathrm{~mm} / \mathrm{L}$ or use of antidiabetic drug, incapacities (Instrumental Activities of Daily Living and Rosow-Breslau functional health scales), cognitive functioning (Mini Mental State score), Center for Epidemiologic Studies-Depression score, APOE genotype). We used this score in different ways. We included it as an adjustment variable in the Cox model. We also calculated hazard ratios within strata defined by thirds of the propensity score distribution. Lastly, we did a matched propensity score analysis. Finally, we estimated hazard ratios in participants who did not report any change in their use of lipid lowering drug (that is, remained a user or non-user) during the first seven years of follow-up.

We did additional analyses to explore complex selection biases. We calculated hazard ratios for death in lipid lowering drug users compared with non-users (all causes of death and deaths from any vascular disease (ICD-10 codes I00-I99)). We estimated hazard ratios for stroke in participants with no previous cardiac event during follow-up, and vice versa. We also estimated the total risk of vascular event (first ever or recurrent) related to lipid lowering drugs during follow-up in the whole Three-City cohort (that is, without excluding participants reporting a history of vascular events at baseline).

We used SAS 9.1 for statistical analyses. We considered a two tailed P value below 0.05 to be statistically significant.

\section{Results}

Among the 7484 participants (mean age 73.9 years, 63\% women), 2048 (27.4\%) reported using lipid lowering drugs (13.5\% statins and $13.8 \%$ fibrates) at baseline. Among fibrate users, 755 (73\%) used fenofibrate; simvastatin $(390 ; 38 \%)$ and pravastatin $(236 ; 23 \%)$ were the most commonly used statins. Five participants used both categories of lipid lowering drugs. Compared with non-users, users of lipid lowering drugs were younger, were more likely to be women, and had a lower level of education, but they otherwise had a higher vascular risk profile (table 1). Lipid lowering drug users had higher blood pressure levels and body mass index; more often had hypertension, diabetes, and cardiac rhythm disorder; and more often used antihypertensive and antithrombotic drugs. Total cholesterol, low density lipoprotein cholesterol, and triglyceride concentrations were significantly lower in users than in non-users, both for statins and fibrates. Compared with users of statins, participants taking fibrates were older, reported lower alcohol consumption, had lower diastolic blood pressure, and took less antithrombotic treatment (table 1). Total cholesterol and triglyceride concentrations were lower in fibrate users than in statin users.

During a mean follow-up of 9.1 years, 732 first ever non-fatal $(n=527)$ or fatal $(n=205)$ cardiovascular events were diagnosed: 440 coronary events (for a total follow-up of 60869 person years) and 292 strokes (for a total follow-up of 61727 person years). Crude incidence rates per 100 person years were 0.72 for coronary events and 0.47 for stroke. Among stroke cases, 227 were ischaemic, 57 haemorrhagic, and eight undefined. Increased risk of stroke or coronary heart disease was associated with classic risk factors: older age, male sex, high blood pressure, diabetes, and high body mass index. Use of antihypertensive or antithrombotic drugs was associated with occurrence of coronary heart disease or stroke. For antihypertensive drugs, hazard ratios were 1.50 (95\% confidence interval 1.24 to 1.82) for coronary heart disease and 1.45 (1.14 to 1.83) for stroke. The risk of coronary heart disease was increased in participants with higher total cholesterol, low density lipoprotein cholesterol, or triglycerides and lower high density lipoprotein cholesterol. We found no association between baseline blood lipid concentrations and the risk of stroke. In particular, the risk of stroke (total or ischaemic) was not increased in participants with elevated triglyceride concentrations.

We found no association between total incidence of vascular events and lipid lowering drug use, either for use of any drug (multivariate model: hazard ratio 0.91 , 0.76 to 1.09 ) or for statins and fibrates examined separately (table 2). However, we observed a very contrasting pattern of association according to the type of event. The risk of coronary heart disease was not lower in lipid lowering drug users (hazard ratio 1.12, 0.90 to 1.40) (table 2). In contrast, we observed a one third decrease in the risk of stroke in lipid lowering drug users (hazard ratio $0.66,0.49$ to 0.90 ) compared with non-users; reduction in stroke risk was similar for the statin and fibrate groups (table 2). Hazard ratios for ischaemic and haemorrhagic stroke in lipid lowering drug users were 0.63 (0.45 to 0.84 ) and 0.72 (0.37 to 1.42). All cause mortality was lower in lipid lowering drug users compared with non-users (hazard ratio 0.87, 0.77 to 0.99 ), but mortality from cardiovascular diseases was not significantly decreased (hazard ratio 0.92, 0.67 to1.25).

Among participants who did not report any change in their use of lipid lowering drugs during the first seven years of follow-up (that is, regular users versus never users), the hazard ratio for stroke in users (any lipid lowering drug) was 0.56 (0.37 to 0.85 ). This analysis did not show significant association between risk of coronary heart disease and use of lipid lowering drugs (hazard ratio 1.22, 0.89 to 1.66 ).

Analyses stratified by age, sex, body mass index, hypertension, systolic blood pressure, and triglyceride 


\begin{tabular}{|c|c|c|c|c|c|c|}
\hline \multirow[b]{2}{*}{ Characteristic } & \multicolumn{6}{|c|}{ Lipid lowering therapy } \\
\hline & No $(n=5436)$ & Yes $(n=2048)$ & Pvalue* & Statins $(n=1007)$ & Fibrates $(\mathrm{n}=1036)$ & Pvalue* \\
\hline Mean (SD) age, years & $74.1(5.6)$ & $73.4(4.8)$ & $<0.001$ & $73.1(4.6)$ & $73.7(4.9)$ & $<0.001$ \\
\hline Women & $62.0(3368)$ & $67.4(1380)$ & $<0.001$ & $67.8(683)$ & $67.2(696)$ & 0.61 \\
\hline \multicolumn{7}{|l|}{ Education: } \\
\hline Medium or low & $61.4(3336)$ & 66.9 (1371) & \multirow{2}{*}{$<0.001$} & $65.3(658)$ & $68.4(709)$ & \multirow{2}{*}{0.22} \\
\hline High & $38.6(2094)$ & $33.1(677)$ & & $34.7(349)$ & $31.6(327)$ & \\
\hline \multicolumn{7}{|l|}{ Smoking: } \\
\hline Never & $62.4(3393)$ & $65.4(1340)$ & \multirow{3}{*}{0.17} & 65.4 (659) & $65.6(680)$ & \multirow{3}{*}{0.99} \\
\hline Past & $31.3(1700)$ & $29.9(613)$ & & $30.2(304)$ & $29.5(306)$ & \\
\hline Current & $6.3(341)$ & $4.6(95)$ & & $4.4(44)$ & $4.8(50)$ & \\
\hline \multicolumn{7}{|l|}{ Alcohol consumption: } \\
\hline Never & $17.5(949)$ & $17.4(355)$ & \multirow{3}{*}{0.008} & $17.1(172)$ & $17.7(183)$ & \multirow{3}{*}{0.07} \\
\hline Past & $2.8(152)$ & $1.7(34)$ & & $1.1(11)$ & $2.2(23)$ & \\
\hline Current & $79.7(4322)$ & $81.0(1657)$ & & $81.8(823)$ & 80.1 (829) & \\
\hline Mean (SD) drinks/day & $1.70(1.6)$ & $1.69(1.6)$ & 0.10 & $1.77(1.7)$ & $1.61(1.6)$ & 0.017 \\
\hline Depressive symptomatology ${ }^{\dagger}$ & $12.8(687 / 5367)$ & $12.8(261 / 2047)$ & 0.58 & $12.3(123 / 1000)$ & $13.2(136 / 1030)$ & 0.51 \\
\hline No disability & $91.0(4906 / 5391)$ & $94.3(1921 / 2037)$ & $<0.001$ & $95(950 / 1000)$ & $93.6(966 / 1032)$ & 0.24 \\
\hline Mean (SD) systolic blood pressure, mm Hg & $146.2(21.9)$ & $147.6(20.9)$ & $<0.001$ & $147.6(20.8)$ & $147.6(21.1)$ & 0.24 \\
\hline Mean (SD) diastolic blood pressure, $\mathrm{mm} \mathrm{Hg}$ & $82.6(11.3)$ & $82.5(10.9)$ & 0.54 & $83.0(10.9)$ & $82.1(10.8)$ & 0.016 \\
\hline Antihypertensive treatment & $41.8(2270 / 5430)$ & $54.1(1107 / 2046)$ & $<0.001$ & $54.7(551)$ & $53.3(552)$ & 0.37 \\
\hline Hypertension $\neq$ & $74.5(4044 / 5428)$ & $79.7(1633 / 2048)$ & $<0.001$ & 79.7 (803) & $79.6(825)$ & 0.51 \\
\hline Cardiac rhythm disorder & $13.1(696 / 5313)$ & $14.6(291 / 1993)$ & 0.032 & $15.6(153 / 981)$ & $13.7(138)$ & 0.13 \\
\hline Antithrombotic treatment & $13.1(659 / 5030)$ & $18.5(379 / 2048)$ & $<0.001$ & $21.6(217 / 1005)$ & $15.5(160 / 1032)$ & $<0.001$ \\
\hline Diabetes§ & $7.2(371 / 5153)$ & $11.9(233 / 1958)$ & $<0.001$ & $10.9(105 / 963)$ & $12.8(126 / 984)$ & 0.13 \\
\hline Mean (SD) body mass index, $\mathrm{kg} / \mathrm{m}^{2}$ & $25.4(4.0)$ & $25.9(4.0)$ & $<0.001$ & $25.8(4.0)$ & $26.0(4.1)$ & 0.18 \\
\hline \multicolumn{7}{|l|}{ Total cholesterol, $\mathrm{mmol} / \mathrm{L}:$} \\
\hline Mean (SD) & $5.97(1.0)$ & $5.60(0.9)$ & $<0.001$ & $5.68(0.9)$ & $5.52(0.8)$ & $<0.001$ \\
\hline$<5.37$ & $26.9(1381)$ & $42.1(818)$ & \multirow{3}{*}{$<0.001$} & $37.8(364)$ & $46.2(452)$ & \multirow{3}{*}{$<0.001$} \\
\hline $5.37-6.18$ & $33.8(1736)$ & $34.8(677)$ & & $36.5(351)$ & $33.2(325)$ & \\
\hline$\geq 6.19$ & $39.2(2013)$ & $23.1(450)$ & & $25.7(247)$ & $20.6(201)$ & \\
\hline \multicolumn{7}{|l|}{ High density lipoprotein cholesterol, mmol/L: } \\
\hline Mean (SD) & $1.63(0.4)$ & $1.64(0.4)$ & 0.16 & $1.64(0.4)$ & $1.63(0.4)$ & 0.56 \\
\hline$<1.41$ & 30.1 (1544) & $29.8(579)$ & \multirow{3}{*}{0.04} & $31.1(299)$ & $28.3(277)$ & \multirow{3}{*}{0.86} \\
\hline $1.41-1.75$ & $34.5(1770)$ & 35.7 (694) & & $33.4(321)$ & $38.0(372)$ & \\
\hline$\geq 1.76$ & 35.4 (1814) & $34.6(672)$ & & $35.6(342)$ & $33.6(329)$ & \\
\hline \multicolumn{7}{|l|}{ Low density lipoprotein cholesterol, mmol/L: } \\
\hline Mean (SD) & $3.78(0.9)$ & $3.40(0.8)$ & $<0.001$ & $3.40(0.9)$ & $3.40(0.7)$ & 0.99 \\
\hline$<3.24$ & $26.1(1336)$ & $45.4(880)$ & \multirow{3}{*}{$<0.001$} & $46.3(444)$ & $44.6(435)$ & \multirow{3}{*}{0.97} \\
\hline $3.24-3.93$ & $33.7(1726)$ & $32.1(623)$ & & $30.6(294)$ & $33.5(327)$ & \\
\hline$\geq 3.94$ & $40.1(2053)$ & $22.5(436)$ & & $23.1(222)$ & $21.8(213)$ & \\
\hline \multicolumn{7}{|l|}{ Triglycerides, $\mathrm{mmol} / \mathrm{L}:$} \\
\hline Geometric mean $(95 \% \mathrm{Cl})$ & $1.14(0.76$ to 1.70$)$ & $1.10(0.70$ to 1.74$)$ & 0.021 & $1.27(0.84-1.93)$ & $0.96(0.62-1.49)$ & $<0.001$ \\
\hline$<0.94$ & $32.1(1646)$ & $38.3(745)$ & \multirow{3}{*}{0.008} & $23.9(230)$ & $52.6(514)$ & \multirow{3}{*}{$<0.001$} \\
\hline $0.94-1.33$ & $35.8(1835)$ & $30.2(587)$ & & $34.0(327)$ & $26.5(259)$ & \\
\hline$\geq 1.34$ & $32.1(1646)$ & $31.5(613)$ & & $42.1(405)$ & $21.0(205)$ & \\
\hline
\end{tabular}

*Pearson $\chi^{2}$ test and analysis of variance for comparison of categorical and continuous characteristics respectively, adjusted for sex, age, and centre.

tWomen scoring $\geq 23$ and men scoring $\geq 17$ on Centre for Epidemiologic Studies-Depression scale.

$\neq B l o o d$ pressure $\geq 140 / 90 \mathrm{~mm} \mathrm{Hg}$ or antihypertensive treatment.

$\S$ Antidiabetic treatment or glycaemia $\geq 7 \mathrm{mmol} / \mathrm{L}$.

concentrations did not show any effect modification by any of these variables (supplementary tables A and B). Adjustment for the propensity score did not modify hazard ratio estimates for lipid lowering drug use (1.09 (0.87 to 1.36 ) for coronary event and 0.65 (0.48 to 0.88 ) for stroke). Analysis stratified by thirds of the propensity score showed that the hazard ratios for stroke were similar in the three strata (supplementary table B). Lastly, risk estimates remained unchanged when treated and untreated participants were matched on propensity score (supplementary table C).
Secondary analyses including participants with a history of a vascular event before entering in the Three-City study also showed a lower risk of total (first ever or recurrent) incident strokes in lipid lowering drug users (statins: hazard ratio 0.70 (0.51 to 0.96 ); fibrates: 0.66 (0.46 to 0.94)). In the same group, hazard ratios for coronary heart disease were 1.45 (1.17 to 1.79) for statins and 1.17 (0.92 to 1.49) for fibrates. The hazard ratio for stroke did not change when participants with a previous cardiac event during follow-up were excluded from the analysis (hazard ratio of stroke in lipid lowering drug users 0.56 (0.41 to 0.77). 


\begin{tabular}{|c|c|c|c|}
\hline \multirow[b]{2}{*}{ Baseline lipid lowering drug use } & \multirow[b]{2}{*}{ No of events } & \multicolumn{2}{|c|}{ Adjusted hazard ratios $(95 \% \mathrm{Cl})$ for vascular events* } \\
\hline & & Model 1 & Model 2 \\
\hline No lipid lowering drug & 545 & Reference & Reference \\
\hline Statins or fibrates & 187 & $0.98(0.83$ to 1.16$)$ & 0.91 (0.76 to 1.09) \\
\hline \multicolumn{4}{|l|}{ Stroke } \\
\hline No lipid lowering drug & 234 & Reference & Reference \\
\hline Statins or fibrates & 58 & $0.71(0.53$ to 0.95$)$ & $0.66(0.49$ to 0.90$)$ \\
\hline Statins & 29 & 0.74 (0.51 to 1.08) & $0.68(0.45$ to 1.01$)$ \\
\hline Statins & 63 & $1.19(0.91$ to 1.56$)$ & 1.13 (0.84 to 1.52$)$ \\
\hline Fibrates & 63 & 1.17 (0.90 to 1.53) & 1.12 (0.84 to 1.49$)$ \\
\hline \multicolumn{4}{|c|}{$\begin{array}{l}\text { *Hazard ratios estimated using Cox proportional hazard models with age as timescale. Model } 1 \text { was adjusted for sex and centre. Model } 2 \text { (multivariable } \\
\text { model) was adjusted as for model } 1 \text { plus diabetes, body mass index, alcohol consumption, smoking, hypertension, cardiac rhythm disorder, } \\
\text { antithrombotic therapy, triglycerides, and low density lipoprotein/high density lipoprotein ratio. } \\
\text { tIncluded hospital admission with definite angina, definite myocardial infarction, definite coronary heart disease death, coronary balloon dilatation, and } \\
\text { coronary artery bypass. }\end{array}$} \\
\hline
\end{tabular}

\section{Discussion}

In a community dwelling population of older people with no known history of vascular events, we found that use of lipid lowering drugs at baseline was associated with a decreased risk of stroke during a mean follow-up of nine years, whereas no protective effect was found for coronary heart disease. This pattern was similar for statin and fibrate use.

\section{Comparison with previous studies}

Previous observational studies have shown that a person's lipid profile is a major risk modifying factor for coronary heart disease, whereas the association between lipid concentrations and stroke is weaker. ${ }^{17}$ Several studies have, however, reported an association between elevated lipid concentrations, particularly triglycerides, and an increased incidence of ischaemic stroke. ${ }^{18} 19$ For intracerebral haemorrhage, an association with low lipid concentrations (both low density lipoprotein cholesterol and triglycerides) has been described. ${ }^{20-22}$ Data from the Cardiovascular Health Study have suggested that among people for whom lipid lowering treatment was recommended, those using a statin had a fourfold lower risk of silent cerebral infarcts compared with non-users. ${ }^{23}$ However, no observational study has ever described a significant association between use of lipid lowering drugs and decreased risk of stroke.

Clinical trials in different populations of patients have extensively evaluated the effects of statins on the incidence of major cardiovascular events. Meta-analyses consistently showed that, compared with placebo, statin treatment reduced the incidence of cardiovascular events by about 20\%; the risk reduction was higher for coronary events than for ischaemic stroke. ${ }^{5}$ Overall, statin treatment had no significant effect on the incidence of haemorrhagic stroke in individual trials, except in a study in which a high dose of atorvastatin was associated with an increased risk of cerebral haemorrhage in patients with a past history of stroke or transient ischaemic attack. ${ }^{24}$ Randomised controlled trials have shown that use of fibrates for secondary prevention of cardiovascular events produced an average 10\% reduction in the incidence of total events, but no consistent effect on the risk of stroke was found. ${ }^{10}$ Some primary prevention trials were conducted in patients at high risk, especially in those with type 2 diabetes. ${ }^{7} 25$ In the ACCORD (Action to Control Cardiovascular Risk in Diabetes) study, simvastatin monotherapy was compared with a combination of simvastatin plus fenofibrate in patients with type 2 diabetes. The combination of statin and fibrate did not reduce the risk of vascular events, compared with simvastatin alone. ${ }^{26}$ In a large meta-analysis focusing on the relation of incident stroke to lipid lowering drug (statins or fibrates) and non-drug (such as diet) interventions, statin treatment was the only intervention that decreased the risk of total stroke. ${ }^{27}$ A recent meta-analysis of individual data from 27 randomised trials concluded that lowering low density lipoprotein cholesterol with statins resulted in a significant reduction in coronary heart disease and stroke. Interestingly, the effect of lowering low density lipoprotein cholesterol was similar in participants with the lowest risk of vascular events and in the highest risk groups. ${ }^{28}$ In these meta-analyses, as in most trials, the mean age of participants was around 60-65 years. PROSPER (Prospective Study of Pravastatin in the Elderly at Risk) was the only trial that focused specifically on people aged 70 to 82 years with a history of risk factors for cardiovascular disease. This trial showed that pravastatin reduced the incidence of major coronary events but not of ischaemic stroke. ${ }^{29}$

\section{Meaning of results and potential for bias}

Our results are at variance with those of randomised controlled trials, as we found that only the risk of 
stroke was decreased in users of lipid lowering drugs, whereas no decrease was observed for risk of coronary heart disease. Could our results be explained by biases? Confounding by indication is a major bias in observational epidemiological studies of drug effects. In real world conditions, the indication for treatment is related to the risk profile of the patient. In this study, such a bias was observed for use of antihypertensive and antithrombotic drugs, as both were associated with an increased risk of stroke. Users of lipid lowering drugs were at higher vascular risk than non-users, and an indication bias would have resulted in an increased risk of stroke, as we observed for other drugs prescribed for reducing the vascular risk (antihypertensive and antithrombotic). Furthermore, use of propensity scores to better take into account all the characteristics associated with use of lipid lowering drugs, including antihypertensive and antithrombotic drug intake, did not modify the estimated association of lipid lowering drug use with the vascular risk. As use of lipid lowering drugs was associated with a $13 \%$ decrease in all cause mortality, a mortality bias cannot be excluded, but it is unlikely that it could completely explain our results. Stroke and coronary heart disease share most vascular factors, including intake of vascular drugs, and it is therefore implausible that confounding by indication, mortality bias, or any other systematic bias would have resulted in an isolated lowering of the risk of stroke. We also explored the possibility that excluding participants with a history of vascular events at baseline could result in an over-estimation of risk reduction. Participants with a past history of vascular events were at higher risk of an incident vascular event during follow-up, and they used lipid lowering drugs more often than did those not reporting past vascular events. However, when we included participants with a past history of vascular events in the analysis, we found similar results. In conclusion, we believe that biases are not the most plausible explanation for the observed decreased risk of stroke associated with lipid lowering drugs.

If biases do not seem to be the main explanation for these results, how could the difference from randomised clinical trials be explained? The community dwelling population of our study consisted of older people with a mean age of 74 (range 65-100) years at inclusion, at low to moderate cardiovascular risk, who experienced a first ever major vascular event at a late age (79.3 years for coronary event and 81.5 years for stroke). These characteristics are different from those of patients who were recruited in clinical trials, in which most patients were younger and had a marked vascular risk. Our study population and the clinical trials' patients also have quite different lipid profiles. Total cholesterol, low density lipoprotein cholesterol, and triglyceride concentrations were much lower, and high density lipoprotein cholesterol was much higher, in the Three-City study than in most clinical trials (table 3). Interestingly, one the strongest reductions in stroke risk was seen in the JUPITER trial in which
Table 3 | Comparison of distribution of lipid concentrations in Three-City study population and in patients included in 14 randomised clinical trials of statins*

\begin{tabular}{lll} 
& \multicolumn{2}{l}{ Distribution (\%) } \\
\cline { 2 - 3 } $\begin{array}{l}\text { Lipids (mmol/L) } \\
\text { Total cholesterol: }\end{array}$ & Three-City study & Clinical trials \\
\hline$\leq 5.2$ & 24.9 & 21.8 \\
\hline$>5.2-6.5$ & 50.8 & 50.7 \\
\hline$>6.5$ & 24.3 & 27.4 \\
\hline Low density lipoprotein cholesterol: & \\
\hline$\leq 3.5$ & 43.5 & 33.4 \\
\hline$>3.5-4.5$ & 40.6 & 41.5 \\
\hline$>4.5$ & 15.9 & 25.1 \\
\hline High density lipoprotein & & \\
\hline$\leq 0.9$ & 1.4 & 35.1 \\
\hline$>0.9-1.1$ & 5.8 & 28.6 \\
\hline$>1.1$ & 92.7 & 36.3 \\
\hline Triglycerides: & & 34.8 \\
\hline$\leq 1.4$ & 71.7 & 29.1 \\
\hline$>1.4-2.0$ & 19.1 & 36.1 \\
\hline$>2.0$ & 9.2 & \\
\hline
\end{tabular}

*From Baigent et al. ${ }^{5}$

patients with a low lipid concentration were recruited. In this trial, the risk of stroke was halved in the statin arm. ${ }^{30}$ Finally, the Three-City study participants reporting a history of lipid disorders at baseline had been diagnosed as having high lipid concentrations for an average of 12 years (data not described). Therefore, many participants had probably been exposed to statins or fibrates for several years before inclusion in the study. We found a larger reduction in the risk of stroke in participants who reported regular use of lipid lowering drugs during follow-up, compared with the whole user group. Both the long term exposure to lipid lowering drugs and the favourable lipid profile of our population of older people with a low to moderate vascular risk could contribute to explaining the observed reduction in stroke incidence among lipid lowering drug users. ${ }^{27-31}$ This interpretation is consistent with the recent opinion that primary prevention with statins could benefit different categories of patients, including those at low risk. ${ }^{32}$

\section{Potential biological mechanisms}

No biological mechanism could easily explain the specific association of lipid lowering drugs with risk of stroke, as well as the absence of difference between statins and fibrates regarding this risk. Both types of drugs have an effect on lipid particles, but the main effect of statins is to reduce low density lipoprotein cholesterol whereas fibrates are more effective in lowering triglyceride concentrations and increasing high density lipoprotein cholesterol concentrations. In addition to their hypolipidaemic properties, both statins and fibrates have pleiotropic effects (anti-inflammatory, antioxidant, antithrombotic, neuroprotective), which can be related to vascular risk. ${ }^{33-36}$ However, no study on the relative contribution of lipid related and non-lipid related properties of statins and fibrates to the prevention of the coronary heart disease and stroke exists that 
could explain our findings. The absence of association between lipid concentrations and stroke risk and the absence of difference between ischaemic and haemorrhagic stroke are both arguments for a possible nonlipid related mechanism.

\section{Strengths and limitations}

Strengths of the study include the prospective data collection, general population setting, large number of participants and outcome events, high quality data on many factors (including medical history, vascular risk factors, lifestyle, and drug use), and validation of cardiovascular events. Validity of data about exposure to lipid lowering drugs was assessed in a previous study in the Three-City study participants. This study showed a very good agreement between self reported use of lipid lowering drugs and claims for reimbursements registered in the French national healthcare insurance database ( $\kappa$ coefficient $0.85,0.84$ to 0.87 ). ${ }^{37}$ Stroke events can be difficult to diagnose with certainty, especially when self reported, but detailed medical records including brain imaging were available for more than $80 \%$ of adjudicated stroke cases. Some stroke cases may also have been missed through our screening procedures. However, a search for stroke cases among participants from the Dijon centre through a stroke register established in this town showed that only nine cases may have been missed, which would give an estimation of 20 cases for the full cohort during the entire follow-up.

Several limitations should also be considered when interpreting our results. Participants in the Three-City study differed somewhat from the general French population: they had higher education and economic status and better cognitive functioning. Overall, they had a healthy lifestyle, in particular dietary habits, which could contribute to reducing their vascular risk. ${ }^{38}$ They might be more compliant with medical prescriptions. However, a major effect of these characteristics on the relation between use of lipid lowering drugs and risk of stroke is unlikely. The possibility that our findings are due either to chance or to residual confounding cannot, however, be ruled out. Lastly, despite the size of the cohort, the study's power was insufficient for detecting small variations in risk of infrequent events. This could explain why the association between lipid lowering drug use and risk of coronary heart disease was not statistically significant.

\section{Clinical implications}

Despite limitations due to voluntary participation, the Three-City study participants are much closer to the general population of older people than are patients included in randomised controlled trials, who are highly selected. Medicines agencies are increasingly concerned about the safety and effectiveness of drugs in real world conditions. The true effectiveness of a drug can differ from the pre-approval evaluation based on clinical trials, because drugs may be used in different patient populations, in different regimens, and for longer periods of time. Our study provides a striking illustration of this concern and supports the need for observational studies in community dwelling population settings. ${ }^{39}$ Our data raise the hypothesis of protection against stroke related to long term use of lipid lowering drugs for primary prevention in older people, with no difference between statins and fibrates. In our population, the incidence of stroke was low (overall, 0.47 per 100 person years) and a $30 \%$ reduction in stroke risk results in a limited number of avoided cases. However, the overall French population is at low risk of stroke, as well as coronary heart disease, and a one third reduction in stroke risk, if confirmed, could have an important effect on public health in other populations.

During the past two decades, the proportion of people taking lipid lowering drugs, especially statins, has increased in most high income countries. Among the people affiliated to the French national healthcare insurance plan, the increase in the prevalence of lipid lowering drug use was much higher over the age of 80 years than in younger elderly people. In French men aged 80-84 years, the proportion of lipid lowering drug users increased from $18 \%$ in 2003 to $25 \%$ in 2012; in women, this proportion increased from $20 \%$ to $26 \%$. A similar increase was observed in people aged 85 years and over. This increase could have contributed to the $13 \%$ decrease in the incidence of stroke in the French population aged 65 years and over during the past decade (www.sante.gouv.fr/les-chiffres-clesde-l-avc).

Contributors: $\mathrm{AA}$, TK, and $\mathrm{CT}$ were responsible for the study concept and design. AA, MLA, SD, CH, and CT acquired the data. All authors were involved in analysis and interpretation of data. AA and CT drafted the manuscript, and all authors revised it critically for important intellectual content. AA is the guarantor.

Funding: The Three-City (3C) Study was conducted under a partnership agreement between the Institut National de la Santé et de la Recherche Médicale (INSERM), the Victor Segalen- Bordeaux II University, and Sanofi-Aventis. The Fondation pour la Recherche Médicale funded the preparation and initiation of the study. The $3 \mathrm{C}$ Study is also supported by the Caisse Nationale Maladie des Travailleurs Salariés, Direction Générale de la Santé, MGEN, Institut de la Longévité, Conseils Régionaux of Aquitaine and Bourgogne, Fondation de France, and Ministry of Research-INSERM Programme "Cohortes et collections de données biologiques," the Fondation Plan Alzheimer. The funding organisations played no role in the design and conduct of the study; in the collection, management, analysis, and interpretation of the data; or in the preparation, review, or approval of the manuscript.

Competing interests: All authors have completed the ICMJE uniform disclosure form at www.icmje.org/coi_disclosure.pdf (available on request from the corresponding author) and declare: support for the submitted work as described above; AA has received within the past three years honorariums for reviewing research projects for the French National Medicines Agency, the Fondation Plan Alzheimer, and the Fondation Bettencourt-Schueller; SD has received research support from the Agence Nationale de la Recherche and from the Fondation Leducq; CT has received research support from Agence Nationale de la Recherche and Fondation Plan Alzheimer for the 3C Study; TK has received within the past two years investigator initiated research funding from the French National Research Agency and the US National Institutes of Health, and has received honorariums from the American Academy of Neurology for educational lectures and from The $B M J$ and Cephalalgia for editorial services.

Ethical approval: The Ethical Committee of the University Hospital of Kremlin-Bicêtre approved the study protocol. Each participant signed an informed consent form.

Transparency declaration: The lead author (the manuscript's guarantor) affirms that the manuscript is an honest, accurate, and 
transparent account of the study being reported; that no important aspects of the study have been omitted; and that any discrepancies from the study as planned have been explained.

Data sharing: No additional data available.

This is an Open Access article distributed in accordance with the Creative Commons Attribution Non Commercial (CC BY-NC 4.0) license, which permits others to distribute, remix, adapt, build upon this work non-commercially, and license their derivative works on different terms, provided the original work is properly cited and the use is non-commercial. See: http://creativecommons.org/licenses/

by-nc/4.0/

1 Fleg JL, Aronow WS, Frishman WH. Cardiovascular drug therapy in the elderly: benefits and challenges. Nat Rev Cardiol 2011;8:13-28.

2 Heart Protection Study Collaborative Group. MRC/BHF Heart Protection Study of cholesterol lowering with simvastatin in 20,536 high-risk individuals: a randomised placebo-controlled trial. Lancet 2002;360:7-22

3 Lewis SJ, Moye LA, Sacks FM, et al. Effect of pravastatin on cardiovascular events in older patients with myocardial infarction and cholesterol levels in the average range: results of the Cholesterol and Recurrent Events (CARE) trial. Ann Intern Med 1998;129:681-9.

4 Hunt D, Young P, Simes I, et al. Benefits of pravastatin on cardiovascular events and mortality in older patients with coronary heart disease are equal to or exceed those seen in younger patients: results from the LIPID trial. Ann Intern Med 2001:134:931-40.

5 Baigent C, Keech A, Kearney PM, et al. Efficacy and safety of cholesterol-lowering treatment: prospective meta-analysis of data from 90,056 participants in 14 randomised trials of statins. Lancet 2005;366:1267-78.

6 Rubins HB, Robins SJ, Collins D, et al. Gemfibrozil for the secondary prevention of coronary heart disease in men with low levels of high-density lipoprotein cholesterol. N Engl J Med 1999;341:410-8.

7 Keech A, Simes RJ, Barter P, et al. Effects of long-term fenofibrate therapy on cardiovascular events in 9795 people with type 2 diabetes mellitus (the FIELD study): randomised controlled trial. Lancet 2005;366:1849-61.

8 Jun M, Foote C, LV J, et al. Effects of fibrates on cardiovascular outcomes: a systematic review and meta-analysis. Lancet 2010;375:1875-84.

9 Remick J, Weintraub H, Setton R, Offenbacher J, Fisher E, Schwartzbard A. Fibrate therapy: an update. Cardiol Rev 2008;16:129-41.

10 Taylor F, Huffman MD, Macedo AF, et al. Statins for the primary prevention of cardiovascular disease. Cochrane Database Syst Rev 2013;1:CD004816

11 Keany JF Jr, Curfman GD, Jarcho JA. A pragmatic view of the new cholesterol treatment guidelines. N Engl I Med 2014;370:275-8.

12 Alperovitch A. Vascular factors and risk of dementia: design of the three-city study and baseline characteristics of the study population. Neuroepidemiology 2003;22:316-25.

13 Lawton MP, Brody EM. Assessment of older people: self-maintaining and instrumental activities of daily living. Gerontologist 1969;9:179-86

14 Radloff LS. The CES-D scale: a self-report depression scale for research in the general population. Appl Psychol Meas 1977;1:385-401.

15 Folstein MF, Folstein SE, McHugh PR. "Mini-Mental State”: a practical method for grading the cognitive state of patients for the clinician. J Psychiatr Res 1975;12:189-98.

16 World Health Organization Collaborating Centre for Drug Statistics Methodology. Guidelines for ATC classification and DDD assignment. WHO, 1996.

17 Canoui-Poitrine F, Luc G, Bard JM, et al. Relative contribution of lipids and apolipoproteins to incident coronary heart disease and ischemic stroke: the PRIME Study. Cerebrovasc Dis 2010;30:252-9.
18 Berger JS, McGinn AP, Howard BV, et al. Lipid and lipoprotein biomarkers and the risk of ischemic stroke in postmenopausal women. Stroke 2012:43:958-66.

19 Labreuche J, Touboul PJ, Amarenco P. Plasma triglyceride levels and risk of stroke and carotid atherosclerosis: a systematic review of the epidemiological studies. Atherosclerosis 2009;203:331-45.

20 Wieberdink RG, Poels MM, Vernooij MW, et al. Serum lipid levels and the risk of intracerebral hemorrhage: the Rotterdam Study. Arterioscler Thromb Vasc Biol 2011;31:2982-9.

21 Bonaventure A, Kurth T, Pico F, et al. Triglycerides and risk of hemorrhagic stroke vs. ischemic vascular events: the Three-City Study. Atherosclerosis 2010;210:243-8

22 Wang X, Dong Y, QiX, Huang C, Hou L. Cholesterol levels and risk of hemorrhagic stroke: a systematic review and meta-analysis. Stroke 2013;44:1833-9.

23 Bernick C, Katz R, Smith NI, et al. Statins and cognitive function in the elderly-the Cardiovascular Health Study. Neurology 2005;65:1388-94

24 Amarenco P, Bogousslavsky J, Callahan A, et al. High-dose atorvastatin after stroke or transient ischemic attack. N Engl / Med 2006;355:549-59.

25 Elkeles RS, Diamond JR, Poulter C, et al. Cardiovascular outcomes in type 2 diabetes. A double-blind placebo-controlled study of bezafibrate: the St. Mary's, Ealing, Northwick Park Diabetes Cardiovascular Disease Prevention (SENDCAP) Study. Diabetes Care 1998;21:641-8.

26 Ginsberg HN, Elam MB, Lovato LC, et al. Effects of combination lipid therapy in type 2 diabetes mellitus. N Engl J Med 2010;362:1563-74.

27 De Caterina R, Scarano M, Marfisi R, et al. Cholesterol-lowering interventions and stroke: insights from a meta-analysis of randomized controlled trials. J Am Coll Cardiol 2010;55:198-211.

28 Cholesterol Treatment Trialists' (CTT) Collaborators. The effects of lowering LDL cholesterol in people at low risk of vascular diseases: meta-analysis of individual data from 27 randomised trials. Lancet 2012;380:581-90

29 Shepherd J, Blauw GJ, Murphy MB, et al. Pravastatin in elderly individuals at risk of vascular disease (PROSPER): a randomised controlled trial. Lancet 2002·360:1623-30.

30 Ridker PM, Danielson E, Fonseca FA, et al. Rosuvastatin to prevent vascular events in men and women with elevated C-reactive protein. N Engl J Med 2008;359:2195-207.

31 Heart Protection Study Collaborative Group, Bulbulia R, Bowman L, et al. Effects on 11-year mortality and morbidity of lowering LDL cholesterol with simvastatin for about 5 years in 20,536 high-risk individuals: a randomised controlled trial. Lancet 2011;378:2013-20.

32 Ridker PM, Wilson PWF. A trial-based approach to statin guidelines. IAMA 2013;310:1123-4

33 Steffens S, Mach F. Anti-inflammatory properties of statins. Semin Vasc Med 2004; 4:417-22

34 Corsini A, Bellosta S, Baetta R, Fumagalli R, Paoletti R, Bernini F. New insights into the pharmacodynamic and pharmacokinetic properties of statins. Pharmacol Ther 1999;84:413-28.

35 Tziomalos K, Athyros VG, Karagiannis A, Mikhailidis DP. Antiinflammatory effects of fibrates: an overview. Curr Med Chem 2009:16:676-84

36 Elisaf M. Effects of fibrates on serum metabolic parameters. Curr Med Res Opin 2002;18:269-76.

37 Noize P, Bazin F, Dufouil C, et al. Comparison of health insurance claims and patient interviews in assessing drug use: data from the Three-City (3C) Study. Pharmacoepidemiol Drug Saf 2009;18:310-9.

38 Barberger-Gateau P, Raffaitin C, Letenneur L, Berr C, Tzourio C Dartigues JF. Dietary patterns and risk of dementia: the Three-City cohort study. Neurology 2007;20:1921-30

39 Sorensen HT, Lash TL, Rothman KJ. Beyond randomized controlled trials: a critical comparison of trials with nonrandomized studies. Hepatology 2006;44:1075-82 